effects in vivo operating through mechanisms other than those concerned with inhibition of viral proliferation, experimental evidence has suggested that these could be: inhibition of tumour cell division, changes in tumour cell surface properties, modulation of lymphocyte functions, enhancement of macrophage phagocytic activity and changes in serum complement level. The relative importance of these effects is still unknown; in some cases interferon might act directly on the tumour cells; in others it seems to modify components of the immune system.

Martin Hirsch of the Massachusetts General Hospital in Boston reported that in chimeric mice (models for human organ and bone marrow transplantation) mouse interferon reduces C-type virus production, lymphoma incidence and the severity of graft-versushost reactivity. $\mathrm{He}$ and Edward De Maeyer, Institut du Radium, Orsay have adso shown that it delays skin graft rejection.

In the 1960 s, a number of clinical trials with synthetic interferon inducers administered to patients were inconclusive in that inducers were found to have both toxicity and limited effectiveness with regard to the level of interferon they elicited in man. The alternative, the large-scale production of exogenous human interferon by cultured cells, was pursued in a number of laboratories, particularly at the Central Public Health Laboratory in Helsinki. Here Kari Cantell has used leukocytes obtained from blood bank buffy coats as interferon producers, and has perfected a system of obtaining high titre interferon preparations suitable for clinical use. Now, with Hans Strander of the Karolinska Institute, he has begun to explore the use of his product in the treatment of a variety of human cancers.

Late in 1974 Strander reported that in a small group of patients with asteogenic sarcoma treated over the past two years surgical removal of the primary tumour, followed by long-term administration of interferon, seemed to have warded off the occurrence of metastases (National Cancer Institute Monograph, in the press). The high titre exogenous interferon he uses seems to be devoid of overt toxicity.

These early results provided the impetus for the workshop, which was designed to review them critically. A careful review by $\mathrm{Dr}$ Strander of his clinical evidence was followed by the report by Alan Rabson (National Cancer Institute) on two independent reviews of the pathology of the treated Scandinavian cases. The originally malignant nature of all the tumours was confirmed, but it was felt that statistical analysis could be based only on that bone tumour type for which solid prog- nostic data exist, agreed by all to be classical osteogenic sarcoma. It was concluded that the rate of recurrence of disease in the form of lung metastases in the cases so selected did appear reduced following interferon administration (with borderling statistical significance because of the small sample size and the relatively shont follow up) when comparied to the rate of recurrence among Swedish historical controls or, for that matter, the generally observed rate of recurrence.

Emil Frei, Director of the Sidney Farber Cancer Center in Boston, then observed that it is not yet clear how the Scandinavian results will compare to those obtainable with recent effective chemotherapy, but that interferon's lack of toxicity could be considered a distinct advantage if it were to have clinical usefulness. It was agreed that the clinical trials in osteogenic sarcoma should be expanded.

The implication of this is that more human interferon must be produced.

A session of the workshop concerned with new methods of interferon production indicated that this expansion of production can be achieved by the application of modern technologies in large scale cell culture. The interferon so produced would still be insufficient for wide use. Thus biochemists must pursue human interferon's purification, sequencing and, ultimately, synthesis,
THERE is much current, interest in alpha-transfer reactions (Nature, 252,$270 ; 1974$ ) because of the information they can give us about alphaparticle clustering in the nuclear surface. There are many reactions that either remove an alpha particle from the nucleus or give an alpha particle to the nucleus, and among these the $\left(\mathrm{d},{ }^{6} \mathrm{Li}\right)$ reaction is particularly convenient because it is relatively easy to produce intense deuteron beams and to detect the emitted ${ }^{6} \mathrm{Li}$ ions.

This reaction has recently been used by the Michigan group (Becchetti et al., Phys. Rev. Lett., 34, $225 ; 1975)$ to make a systematic study of alpha pickup in a range of nuclei from ${ }^{12} \mathrm{C}$ to ${ }^{238} \mathrm{U}$. They irradiated these nuclei with $35 \mathrm{MeV}$ deuterons, and observed the ${ }^{6} \mathrm{Li}$ emitted at the angle corresponding to the maximum in the cross section for $\mathrm{L}=0$ transfer. They found that the cross section falls rather steeply as the target mass increases (roughly as $A_{\mathrm{t}}{ }^{-3}$ ), with superposed fluctuations that are connected with the nuclear shell structure.

To study these variations in more detail, they calculated the cross section for alpha-particle pickup using the distorted wave theory. This contains a factor $S_{u}$ that is a measure of

\section{Alpha-transfer reactions}

from P. E. Hodgson

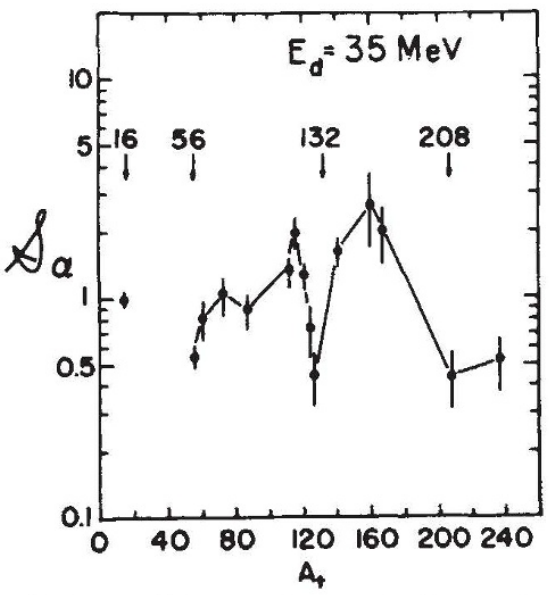

Alpha-particle spectroscopic factor $S$ obtained from analyses of $\left(d,{ }^{6} \mathrm{Li}\right) \mathrm{re}$ actions normalised to unity at ${ }^{16} \mathrm{O}$ and plotted as a function of target mass.

the probability of finding an alpha particle in the target nucleus. The resulting values of $S_{\alpha}$, normalised to unity for ${ }^{16} \mathrm{O}$, are shown as a function of $A$ in the figure.

The fluctuations of $S_{\alpha}$ with $A$ can be correlated with the nuclear shell structure in a very simple way. The minima correspond to nuclei with closed neutron or proton shells, and the maxima correspond to nuclei with open shells. Thus the maximum around $A=110$ corresponds to open neutron shells and that around 160 to open neutron and proton shells.

These results are closely parallel to those found by Milazzo-Colli and Braga-Marcazzan in their studies of (p, $\alpha)$ and $(\mathrm{n}, \alpha)$ reactions (Nature, 245,$12 ; 1973$ ), and of alpha decay. The probability of alpha emission is given by the product of the probability of finding an alpha particle on the nuclear surface and the probability that it can penetrate through the potential barrier of the emitting nucleus. Analysis of data on many spontaneous alpha decays gave the variation of the alpha preformation probability with $A$ (Bonetti and Milazzo-Colli, Phys. Lett., 49B, 17; 1974). Their result was similar to that shown in the figure and in particular showed a marked minimum in the region of the doubly closed shell nucleus ${ }^{208} \mathrm{~Pb}$.

Taken together, these results provide useful additional evidence concerning the probability of finding alpha particles on the nuclear surface. 